Review Article

\title{
Systematic Review of Compound Danshen Dropping Pill: A Chinese Patent Medicine for Acute Myocardial Infarction
}

\author{
Jing Luo, ${ }^{1}$ Hao $\mathrm{Xu}^{2}$ and Keji Chen ${ }^{2}$ \\ ${ }^{1}$ Graduate School, Beijing University of Chinese Medicine, Beijing 100029, China \\ ${ }^{2}$ Cardiovascular Diseases Center, Xiyuan Hospital, China Academy of Chinese Medical Sciences, Beijing 100091, China \\ Correspondence should be addressed to Hao Xu; xuhaotcm@hotmail.com and Keji Chen; keji_chen@yahoo.com
}

Received 26 February 2013; Accepted 23 May 2013

Academic Editor: Myeong Soo Lee

Copyright (c) 2013 Jing Luo et al. This is an open access article distributed under the Creative Commons Attribution License, which permits unrestricted use, distribution, and reproduction in any medium, provided the original work is properly cited.

\begin{abstract}
Objective. This paper systematically evaluated the efficacy and safety of compound Danshen dropping pill (CDDP) in patients with acute myocardial infarction (AMI). Methods. Randomized controlled trials (RCTs), comparing CDDP with no intervention, placebo, or conventional western medicine, were retrieved. Data extraction and analyses were conducted in accordance with the Cochrane standards. We assessed risk of bias for each included study and evaluated the strength of evidence on prespecified outcomes. Results. Seven RCTs enrolling 1215 patients were included. CDDP was associated with statistically significant reductions in the risk of cardiac death and heart failure compared with no intervention based on conventional therapy for AMI. In addition, CDDP was associated with improvement of quality of life and impaired left ventricular ejection fraction. Nevertheless, the safety of CDDP was unproven for the limited data. The quality of evidence for each outcome in the main comparison (CDDP versus no intervention) was "low" or "moderate." Conclusion. CDDP showed some potential benefits for AMI patients, such as the reductions of cardiac death and heart failure. However, the overall quality of evidence was poor, and the safety of CDDP for AMI patients was not confirmed. More evidence from high quality RCTs is warranted to support the use of CDDP for AMI patients.
\end{abstract}

\section{Introduction}

Acute myocardial infarction (AMI) is a serious type of coronary heart disease (CHD) and a major cause of death worldwide with an estimated annual incidence rate of seven million people [1]. As a result of coronary artery thrombotic occlusion from plaques rupture or erosion, AMI usually leads to death if complicated by severe heart failure, malignant ventricular arrhythmia, or cardiac rupture $[1,2]$. Despite the application of percutaneous coronary intervention (PCI) and conventional western medicine, AMI patients remain at certain risk of in-hospital death and complications as well as recurrent acute cardiovascular events [2-4]. With more and more clinicians successfully applied traditional Chinese medicine (TCM) in CHD prevention and treatment based on conventional therapy, the effects of TCM for CHD have drawn more and more attention [5-8].

Compound Danshen dropping pill (CDDP, also known as the "Dantonic Pill"), a Chinese oral patent medicine, has been widely used for cardiovascular diseases, including AMI, in China and some Asia countries. The phase II clinical trial of CDDP to treat chronic stable angina (http://clinicaltrials.gov/, NCT00797953) had been completed in the United States in 2010. Moreover, this drug has been approved by the Australian Therapeutic Goods Administration for use and is widely available in Australia [9]. CDDP consists of three compositions, namely, Radix Salviae Miltiorrhizae, Radix Notoginseng, and Borneolum Syntheticum. These compositions and their pharmacological actions [10-15] are listed in Table 1 with common, pinyin, and Latin names. Previous pharmacologic studies and randomized clinical trials have indicated the potential benefit of CDDP for patients with AMI [16-21]. Recent systematic reviews [22-24] also revealed potential benefits of CDDP for angina pectoris. The efficacy and safety of CDDP for AMI, however, have not been systematically evaluated. The aim of this study was to assess the efficacy and safety of CDDP on the treatment of AMI patients. 
TABLE 1: Compositions of compound Danshen dropping pill.

\begin{tabular}{|c|c|c|c|}
\hline Common name & Pinyin name & Latin name & Pharma. actions \\
\hline Danshen root & Danshen & $\begin{array}{l}\text { Radix Salviae } \\
\text { Miltiorrhizae }\end{array}$ & $\begin{array}{l}\text { Dilates coronary vessels and antimyocardial ischemia inhibit platelet aggregation } \\
\text { and thrombosis, decrease cholesterol and endothelial damage, scavenge free } \\
\text { radicals, antilipid peroxidative, and antiatherosclerosis, and reduce myocardial } \\
\text { ischemia-reperfusion injury, anti-inflammatory }[10,11] \text {. }\end{array}$ \\
\hline Sanchi root & Sanqi & $\begin{array}{l}\text { Radix } \\
\text { Notoginseng }\end{array}$ & $\begin{array}{l}\text { Dilates blood vessel increases blood platelet number to promote hemostasis, } \\
\text { inhibits platelet aggregation and thrombosis, and reduces viscosity of whole blood, } \\
\text { decreases the heart rate and myocardial ischemia-reperfusion injury, inhibits } \\
\text { proliferation of vascular smooth muscle cell, decreases cholesterol and } \\
\text { antiatherosclerosis, antioxidation }[12,13] \text {. }\end{array}$ \\
\hline Borneol & Bingpian & $\begin{array}{l}\text { Borneolum } \\
\text { Syntheticum }\end{array}$ & $\begin{array}{l}\text { Analgesia and sedation boost other drugs' bioavailability, anti-inflammatory, and } \\
\text { decreases the heart rate and myocardial oxygen consumption }[14,15] .\end{array}$ \\
\hline
\end{tabular}

\section{Methods}

2.1. Inclusion and Exclusion Criteria. Randomized controlled trials (RCTs) comparing CDDP with no intervention, placebo, or conventional western medicine were sought regardless of their publication status. Participants of any gender, age, or ethnic origin with AMI meeting with one of the past or current definitions of AMI [25-29] were included. Those without description of diagnostic criteria but stated patients with definite AMI were also considered. Quasirandomized trials and animal experiments were excluded. Trials with CDDP as adjunctive therapy or with duration less than four weeks were also excluded.

Primary outcomes consisted of all-cause mortality, cardiac mortality, recurrent myocardial infarction (RMI), and revascularization, including PCI and coronary artery bypass graft (CABG). Secondary outcomes included heart failure, readmission, left ventricular ejection fraction (LVEF), recurrent angina, adverse events and health-related quality of life measured by a validated tool.

\subsection{Source of Literature and Search Strategy}

2.2.1. Electronic Searches. We searched the following databases up to October 2012 for the identification of RCTs both published and unpublished: Pubmed, The Cochrane Library, Chinese Biomedical Database (CBM), Chinese VIP Information (VIP), China National Knowledge Infrastructure $(\mathrm{CNKI})$, Wanfang Databases, China Proceedings of Conference Full-text Database (CPCD), Chinese Doctoral Dissertations Full-text Database (CDFD), and Chinese Master's Theses Full-text Database (CMFD). Search strategy in Table 2 was used in The Cochrane Library and adapted appropriately for other databases.

In addition, we searched databases of ongoing trials: ClinicalTrials.gov (http://clinicaltrials.gov/) and Current Controlled Trials (http://www.controlled-trials.com/).

2.2.2. Additional Searches. We also searched the reference lists of studies included in this systematic review and of other relevant reviews to identify missing relevant articles.
TABLE 2: Search strategy for the Cochrane library.

\begin{tabular}{l}
\hline Strategy \\
\hline No. 1 Danshen pill \\
No. 2 salvia pill \\
No. 3 compound Danshen \\
No. 4 compound salvia \\
No. 5 composite Danshen \\
No. 6 composite salvia \\
No. 7 Dantonic Pill \\
No. 8 CDDP \\
No. 9 CSDP \\
No. 10 FFDS \\
No. 11 myocardial infarction $[\mathrm{MeSH}]$ \\
No. 12 coronary disease $[\mathrm{MeSH}]$ \\
No. 13 coronary artery disease $[\mathrm{MeSH}]$ \\
No. 14 acute coronary syndrome $[\mathrm{MeSH}]$ \\
No. 15 myocardial infarct \\
No. 16 AMI \\
No. 17 MI \\
No. 18 acute coronary syndrome \\
No. 19 (1, $2,3,4,5,6,7,8,9$, or 10$)$ \\
No. 20 (11, $12,13,14,15,16,17$, or 18$)$ \\
No. 21 (19 and 20 )
\end{tabular}

2.3. Study Identification and Data Extraction. Two authors (Jing Luo, Hao Xu) independently screened the titles and abstracts of references for potentially relevant RCTs. Full texts of potentially eligible articles were retrieved for further identification according to the inclusion and exclusion criteria. Any disagreement was resolved by consensus.

Two authors (Jing Luo, Hao Xu) independently extracted data using a preset data extraction form. Characteristics of RCTs including methods, participants, interventions, comparisons, and outcomes were extracted. We obtained missing information from the original authors whenever possible and resolved any disagreement through discussion or consulting the third author (Keji Chen). 
2.4. Assessment of Risk of Bias and Quality of Evidence. Two authors (Jing Luo, Hao Xu) independently assessed the methodological quality of each of the included studies using the Cochrane "risk of bias" criteria [30], which covers the following items: random sequence generation, allocation concealment, blinding of participants and personnel, blinding of outcome assessment, incomplete outcome data, selective reporting, and other bias. Disagreements were resolved by consensus. For each item, a low risk was considered when we judged a "Yes," conversely, a "No" for a high risk, and otherwise for an unclear risk.

We also evaluated the quality of evidence of each outcome using the Grading of Recommendations Assessment, Development and Evaluation (GRADE) approach [31], as recommended by the Cochrane Collaboration. Patient important outcomes in the main comparison were judged across five factors: limitations in study design and execution, inconsistency of results, indirectness of evidence, imprecision, and publication bias. Accordingly, we graded the quality of evidence in this review as very low, low, moderate, or high.

2.5. Data Analysis. We used RevMan 5.1 software for data analyses. Studies were stratified by the different types of comparisons. We performed intention-to-treat analysis (ITT) for dichotomous data and presented outcome data as risk ratio (RR) with corresponding 95\% confidence interval (CI). We calculated mean difference (MD) with its $95 \%$ CI for continuous outcomes. Fixed effect model was used to analyze data with low heterogeneity $\left(I^{2} \leq 50 \%\right)$; random effects model was applied if heterogeneity is significant $(50 \%<$ $\left.I^{2}<75 \%\right)$. Results were not pooled for data with high heterogeneity $\left(I^{2} \geq 75 \%\right)$ [32], in which case we explored potential causes of heterogeneity by conducting subgroup analyses based on the characteristics of intervention (dosage, duration) and the types of conventional therapy (PCI versus thrombolysis). We also performed sensitivity analyses on studies with lower methodological quality, in order to investigate whether the inclusion of such studies altered the conclusion of the meta-analysis. Possible publication bias was checked using funnel plots when the number of included studies of any particular outcome is greater than eight.

\section{Results}

3.1. Study Identification. A total of 564 references were found according to search strategy, of which 261 were excluded for duplicates among databases. After screening the abstract, we excluded 231 articles. 72 potentially eligible studies were retrieved for further identification, of which 65 were excluded because they did not meet the prespecified inclusion criteria described in the methods. At last, seven eligible RCTs [1921, 33-36] were included. No ongoing trial was found. Please refer to Figure 1 for a more detailed illustration of the data screening process.

3.2. Description of Included Studies. The characteristics of the included seven studies [19-21, 33-36] are summarized in Table 3. Each of the studies was conducted in China. One postgraduate dissertation [35] was unpublished in 2010, and the others were published from 2006 to 2011. One study [19] was of multicenter design, but the others were of single centre trials.

The number of participants in the individual study ranged from 45 to 500, with a total of 1215 in this review (583 in intervention groups and 632 in control groups). There were 863 males and 352 females included in the review, with mean age, where given $[19,20,34-36]$, ranging from 52 to 66 years. All of the participants were diagnosed with AMI by different diagnostic criteria: two studies $[20,21]$ used the WHO diagnostic criteria; one study [33] used ACC/AHA diagnostic criteria; four studies [19, 34-36] without specified diagnostic criteria but mentioned "patients with AMI were eligible to include." Two studies $[19,35]$ only included patients with ST-elevation myocardial infarction (STEMI), one study excluded AMI without Q wave [21], and the others did not introduce the types of AMI (four studies) [20, 33, 34, 36].

All participants in the intervention groups were treated with CDDP, 10 pills three times a day (tid) orally based on conventional therapy since the day of diagnosis $[19,20,33-$ 36]. Only one study [21] began the CDDP treatment four to five weeks later after diagnosis and changed the dosage from 10 pills tid to five pills tid after 60 days of treatment. The duration of treatment was mainly as same as the length of follow up, ranging from four weeks to 12 months. One study [21] was designed as three groups with two comparisons including CDDP versus no intervention and CDDP versus propranolol. Six studies consisted of two groups (one study [36] compared CDDP with placebo and the others $[19,20,33-$ 35] focused on CDDP compared with no intervention). In total, there were three comparisons in the review: (1) CDDP plus conventional therapy versus conventional therapy (six studies) [19-21, 33-35]; (2) CDDP plus conventional therapy versus placebo plus conventional therapy (one study) [36]; (3) CDDP plus conventional therapy versus propranolol plus conventional therapy (one study) [21].

Five studies [19-21, 35, 36] reported mortality including all-cause mortality (four studies) [19-21,35] and cardiac mortality (three studies) $[21,35,36]$. Two studies provided numerical information on RMI $[21,36]$, but the data could not be pooled for the different comparisons. Four studies [19-21, $36]$ reported heart failure. Three studies $[19,20,36]$ provided the number of patients having recurrent angina. Besides the incidence of readmission and adverse events (narrative introduction), one study [36] also assessed the QOL by questionnaire score, and the questionnaire was designed referring to Treatment of Mild Hypertension Study (TOMHS) and Medical Outcomes Study 36-Item Short- Form Health Survey (SF-36). Five studies $[19,21,33,34,36]$ assessed the LVEF with the aim of evaluating the heart function. None of the included studies mentioned revascularization.

\subsection{Quality of Included Studies}

3.3.1. Risk of Bias in Included Studies. Risk of bias summaries for each outcome in the included RCTs at the study level are presented in Figures $2-10$. No study was felt to have a low 


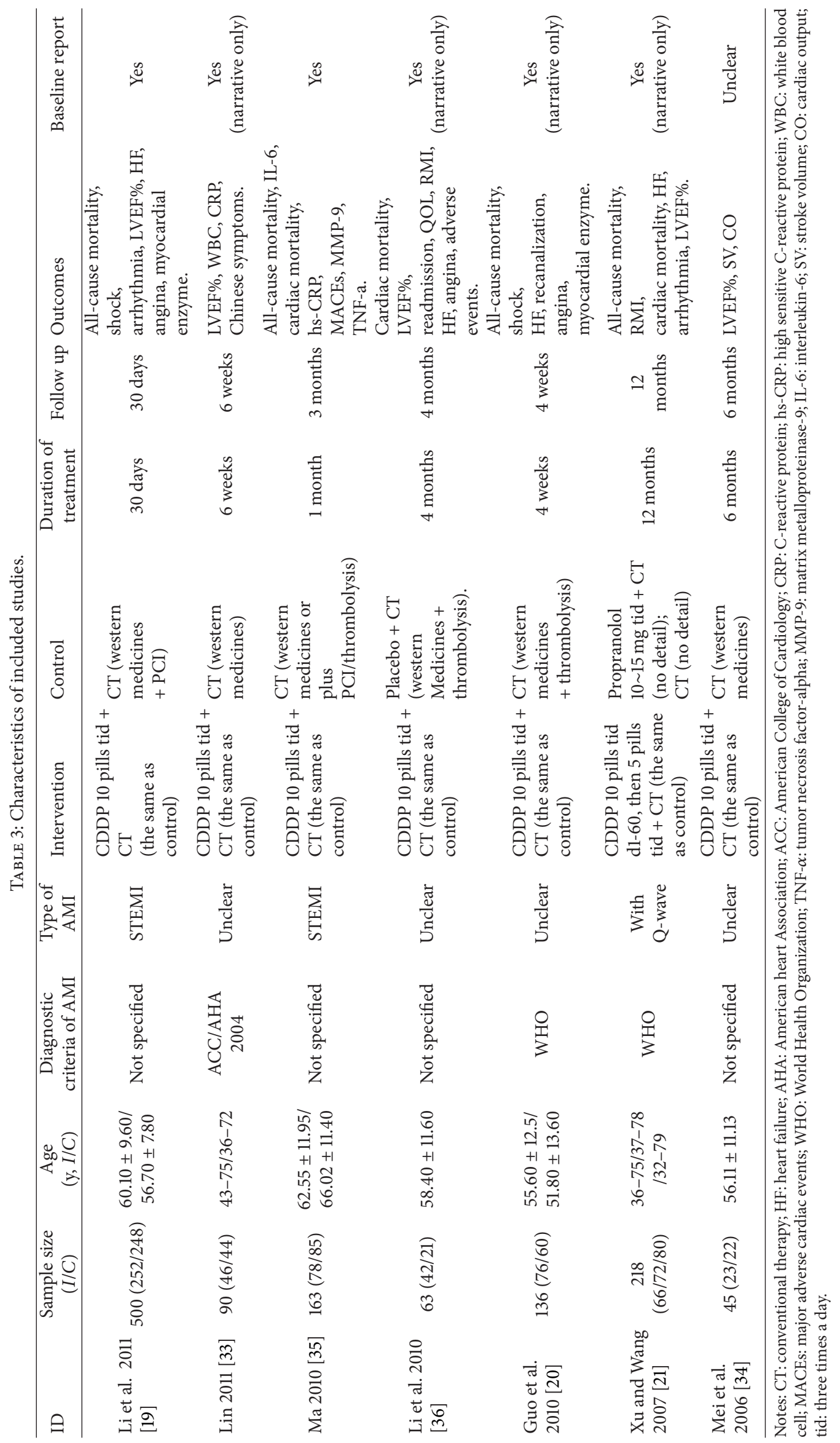




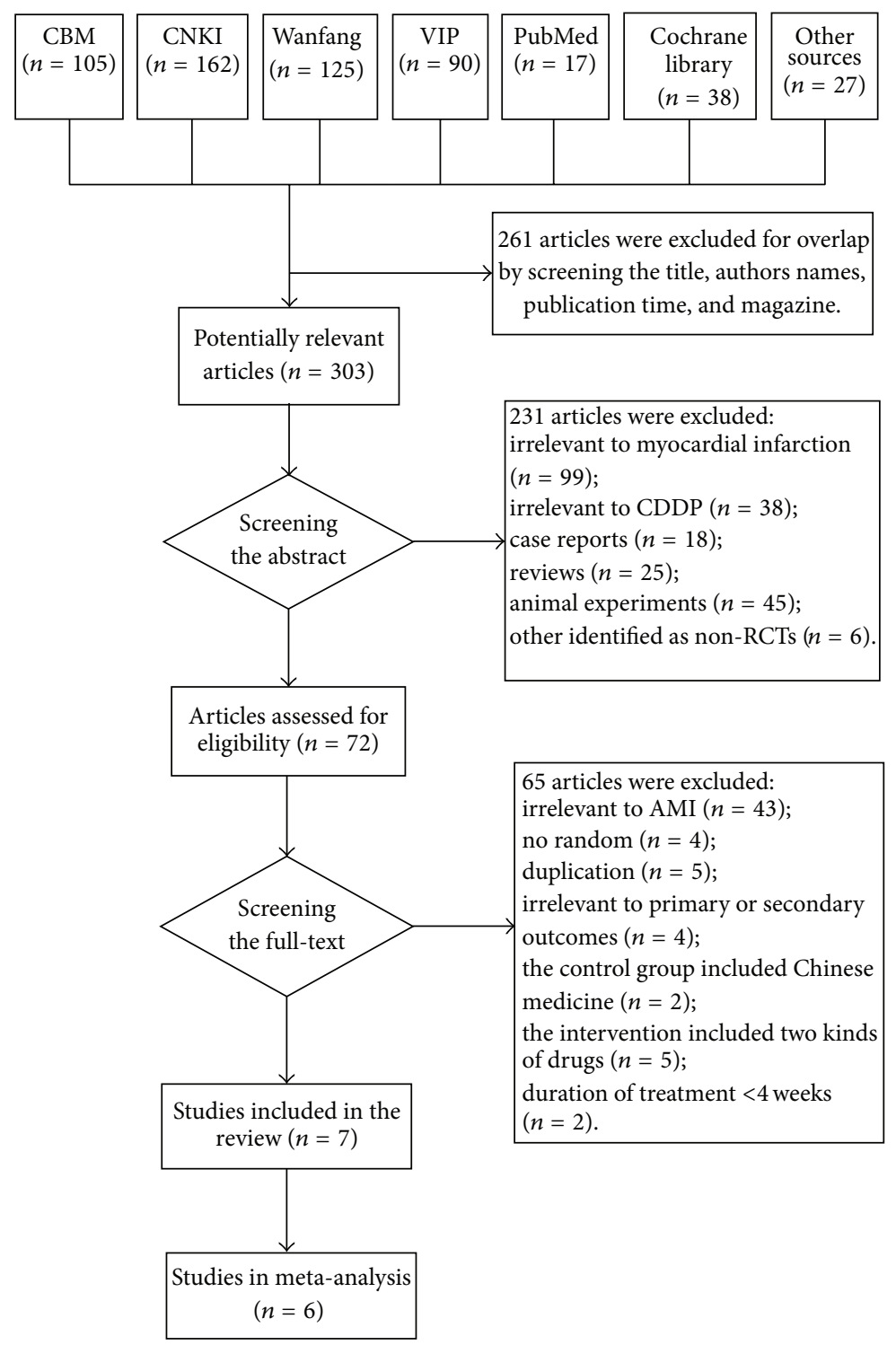

FIGURE 1: Flow chart of study search and identification.

risk of bias. Of the seven studies, one [35] introduced the random sequence being generated from a random number table, and the others just mentioned "patients were randomly allocated" without the method of randomization. Only one study [19] reported allocation concealment. None of the studies described blinding of participants and personnel although one study [36] used placebo. All of the studies did not report blinding of outcome assessment. Neither withdrawals nor losses to follow up were reported in the studies. One study [19] had incomplete outcome data. Five studies $[20,21,33,35,36]$ reported the comparability of the baseline among groups, but four of them did not provide baseline data $[20,21,33,36]$. The multicenter study [19], with other similar baselines, reported that the rate of diabetes patients in the intervention group was higher than the control group. In addition, no study mentioned prior sample size estimation or ITT analysis for any outcome.
After we contacted with the original authors by telephone and email, only one author [19] told us that there was no blinding of participants or personnel in their study, and the randomization was designed by public health statistics teaching and research section of Tianjin Medical University; he did not know any other details. In fact, due to a number of unsuccessful contacts and some unclear or unavailable replies, most of our questions were not resolved.

3.3.2. Quality of Evidence in Included Studies. The quality of evidence for each outcome in the main comparison (CDDP versus no intervention) was ranged from "low" to "moderate" (Table 4). Quality assessment of the evidence in accordance with the GRADE approach showed some limitations of the study design and execution, inconsistency, indirectness, and imprecision. Due to the low number of included studies for each outcome, we could not create funnel plots to detect 


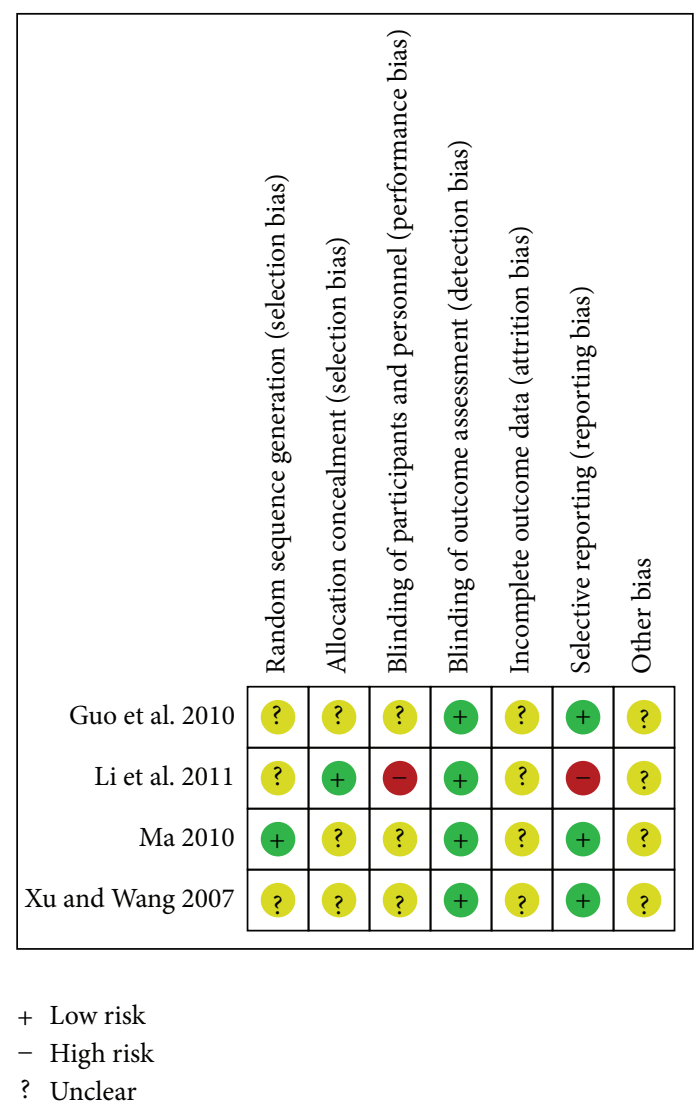

FIGURE 2: Risk of bias summary—all-cause mortality.

publication bias. For each outcome, there were one or two serious limitations among the five factors. For example, because of the serious risk of bias and imprecision for allcause mortality in the main comparison, we downgraded the quality rating by two levels, thus the quality of evidence for this outcome was low. The quality of evidence was moderate for cardiac mortality and heart failure, low for all-cause mortality, RMI, recurrent angina, and LVEF.

\subsection{Effect of Interventions (Table 5 to Table 7)}

3.4.1. All-Cause Mortality (Table 5). Four studies [19-21, 35] reported all-cause mortality in two different comparisons. Meta-analysis of the four studies showed no statistically significant difference in the risk of all-cause death between CDDP and no intervention (RR 0.65 ; 95\%CI 0.37 to 1.14 ; $n=945$ ). Sensitive analysis, excluding the lower quality study [19], found that CDDP was associated with a statistically significant reduction in the risk of all-cause death compared with no intervention without heterogeneity (RR $0.51 ; 95 \% \mathrm{CI}$ 0.27 to 0.98 ; three studies, $n=445 ; I^{2}=0 \%$ ) $[20,21,35]$. A single study reported that there was no statistical difference in reducing all-cause mortality between CDDP and propranolol on the basis of conventional therapy (RR $0.65 ; 95 \%$ CI 0.16 to $2.63 ; n=138)[21]$. The associated risk of bias is presented

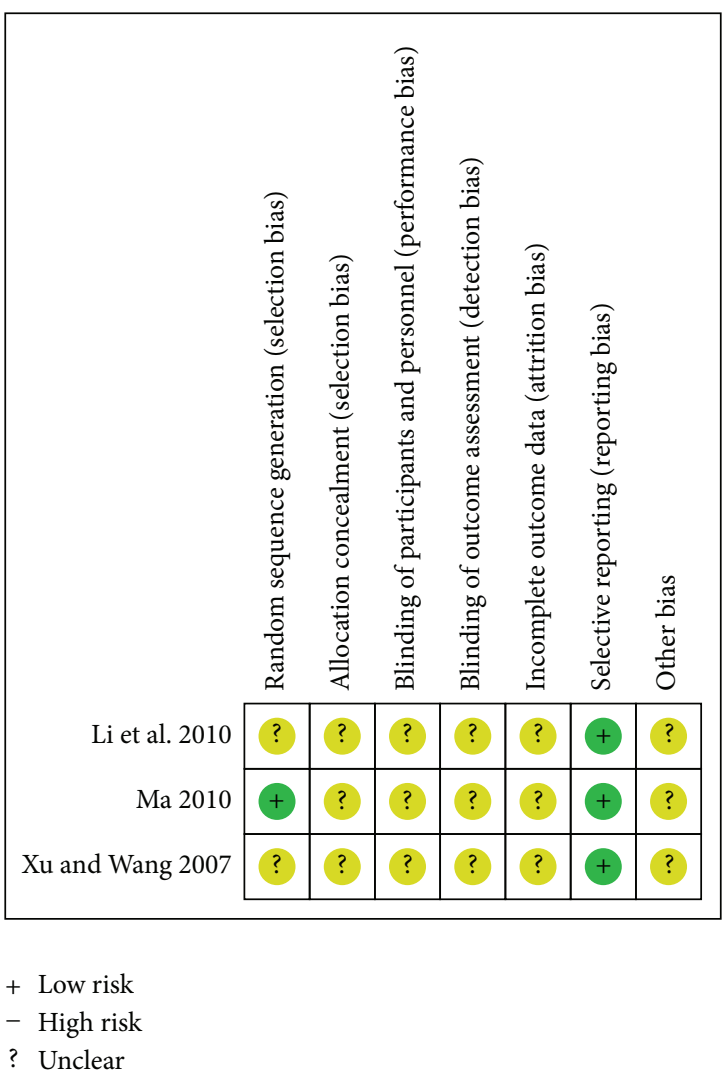

FIGURE 3: Risk of bias summary—cardiac mortality.

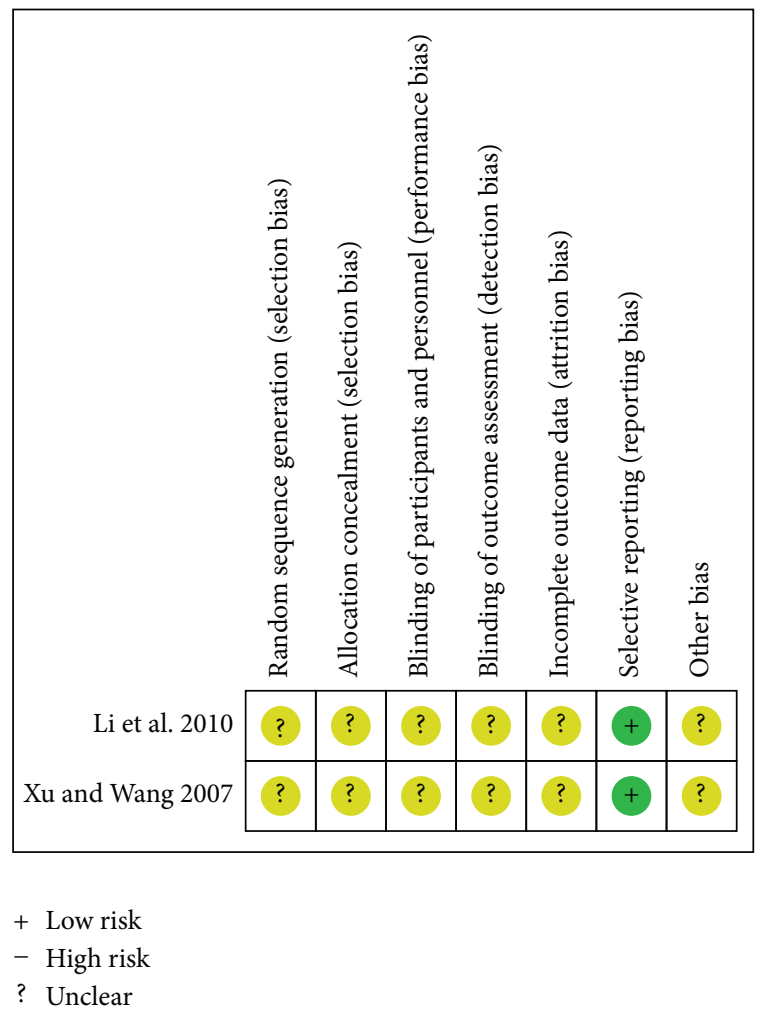

FIGURE 4: Risk of bias summary-recurrent myocardial infarction. 


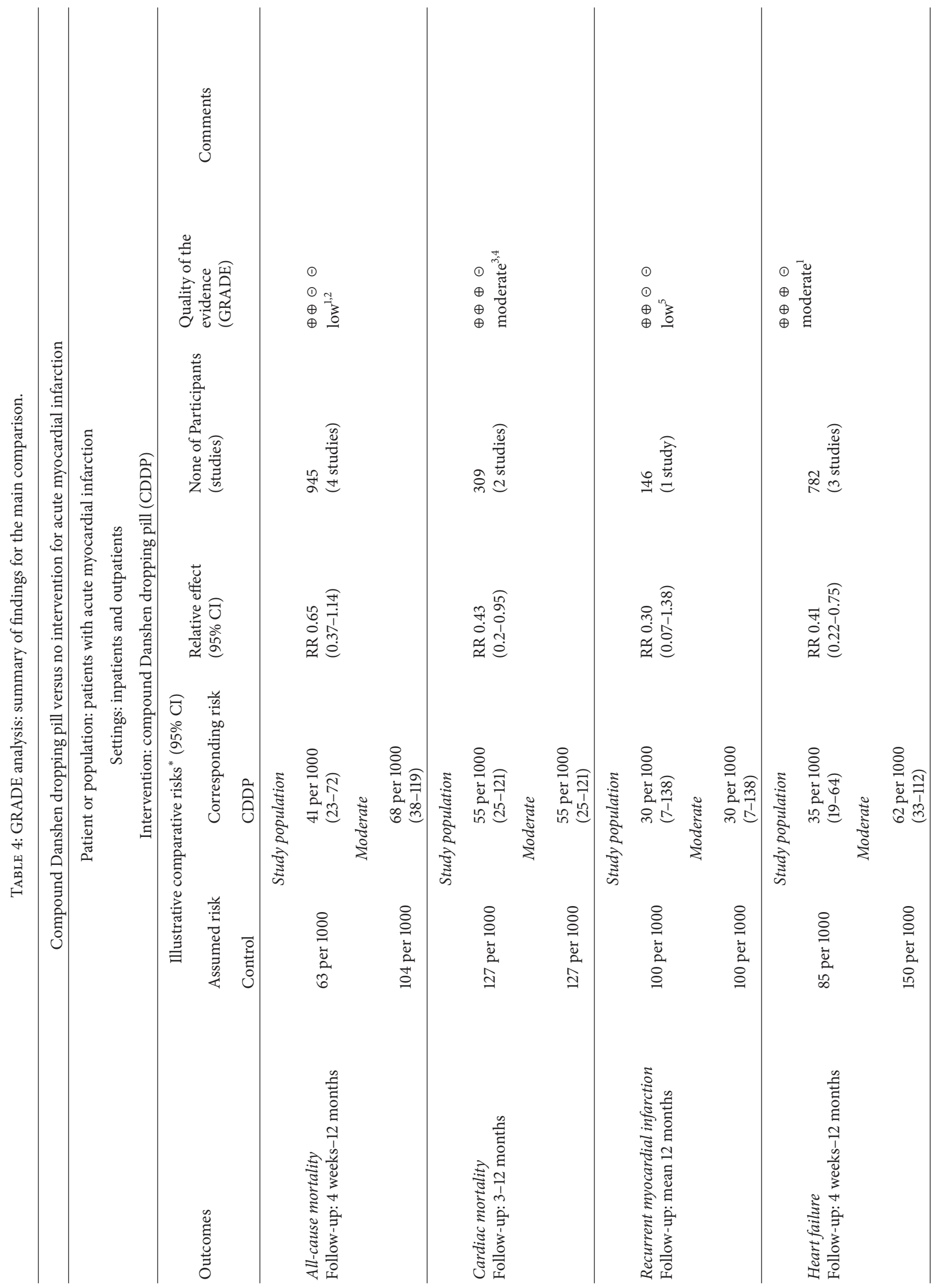




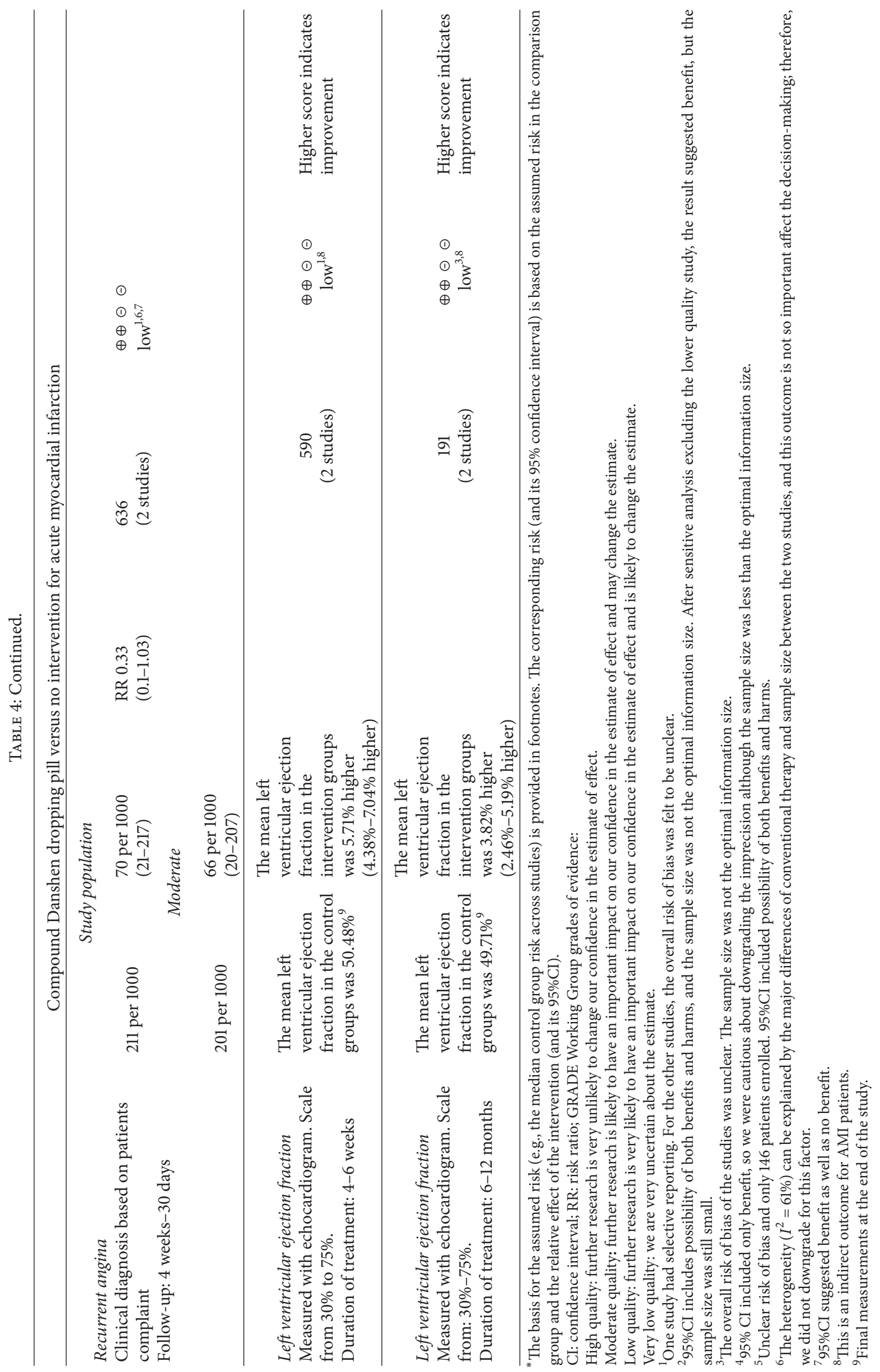




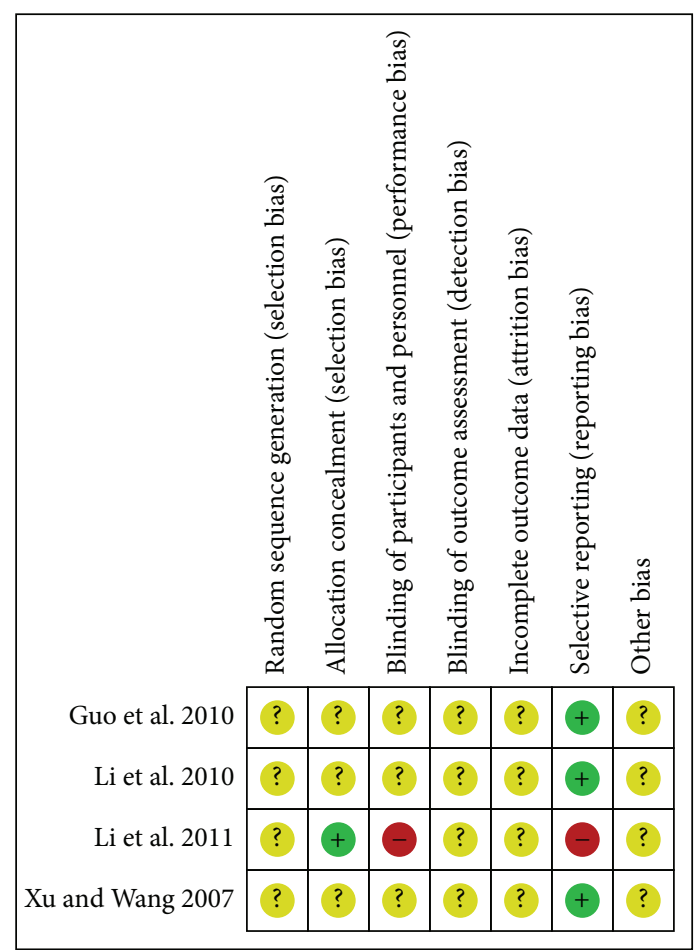

+ Low risk

- High risk

? Unclear

FIGURE 5: Risk of bias summary-heart failure.

in Figure 2. The quality of evidence in the main comparison (CDDP versus no intervention) was low (Table 4).

3.4.2. Cardiac Mortality (Table 5). Three studies [21, 35, 36] assessed cardiac mortality in three different comparisons. Meta-analysis of two studies [21,35] showed that CDDP was associated with a statistically significant reduction in the risk of cardiac death compared with no intervention without heterogeneity (RR 0.43 ; $95 \% \mathrm{CI} 0.20$ to $0.95 ; n=309 ; I^{2}=$ $0 \%)$. Compared with placebo on the basis of conventional therapy, CDDP had no statistically significant advantage in reducing cardiac mortality (RR $0.50 ; 95 \% \mathrm{CI} 0.03$ to 7.60 ; one study, $n=63$ ) [36]. A single study reported a similar result between CDDP and propranolol (RR 0.81; 95\%CI 0.17 to 3.76; $n=138$ ) [21]. Figure 3 presents the associated risk of bias. The quality of evidence in the main comparison (CDDP versus no intervention) was moderate (Table 4).

3.4.3. Recurrent Myocardial Infarction (Table 5). Two studies $[21,36]$ reported RMI in three different comparisons. None of the comparisons, however, presented a statistically significant difference in the risk of RMI: CDDP versus no intervention (RR 0.30; 95\%CI 0.07 to 1.38 ; one study, $n=146$ ) [21]; CDDP versus placebo (RR 0.50; 95\%CI 0.11 to 2.27 ; one study, $n=63$ ) [36]; CDDP versus propranolol (RR 0.73; 95\%CI 0.13 to 4.22 ; one study, $n=138$ ) [21]. The associated risk of

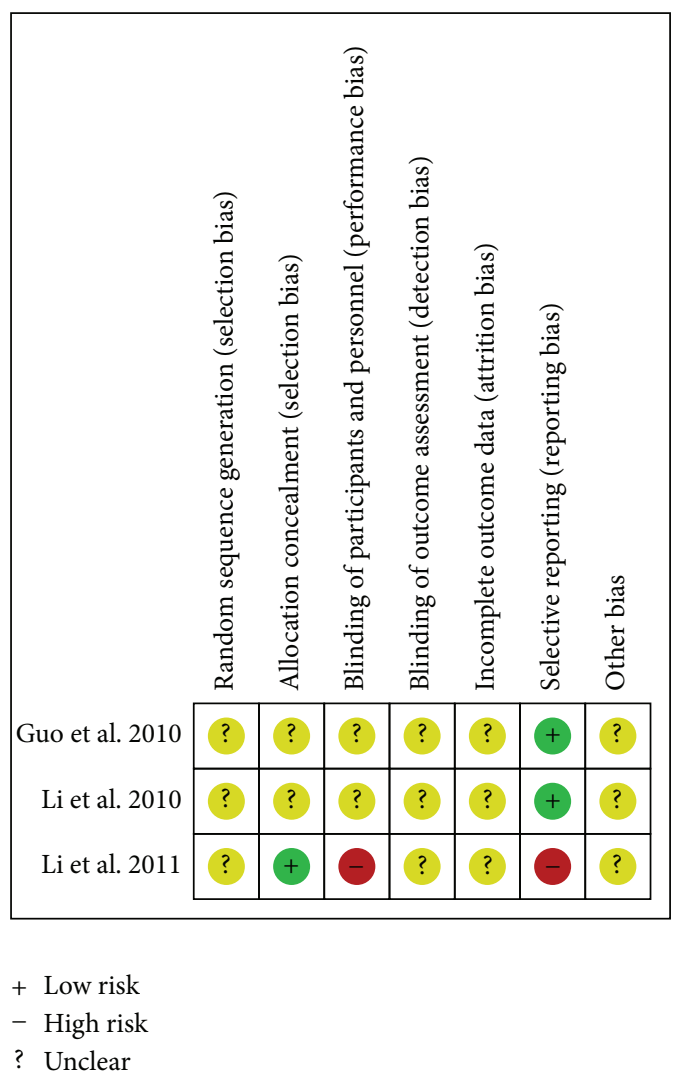

FIGURE 6: Risk of bias summary-recurrent angina.

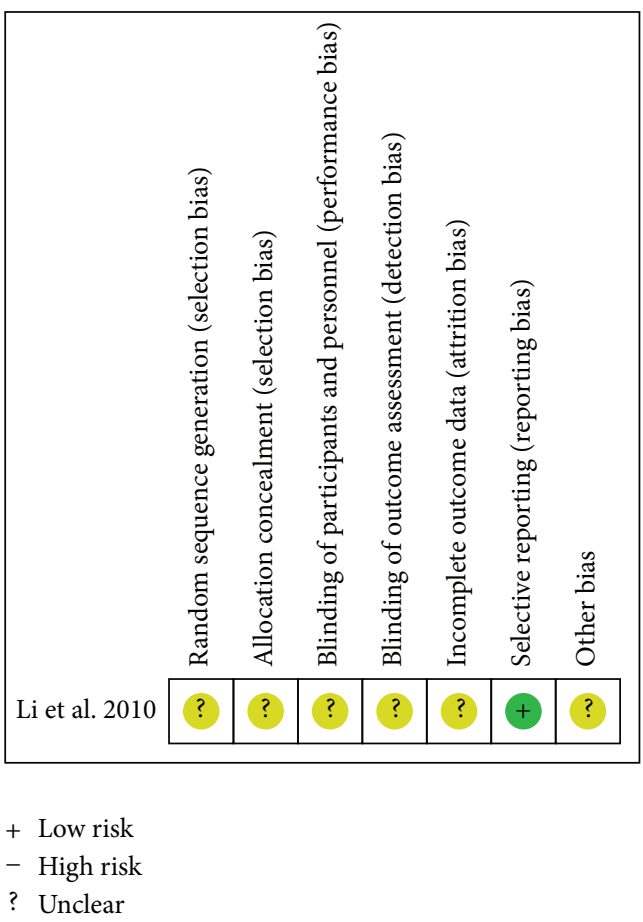

FIGURE 7: Risk of bias summary—readmission. 
TABle 5: Analyses of primary outcomes.

\begin{tabular}{|c|c|c|c|c|c|}
\hline Outcomes (comparisons) & Treatment $(n / N)$ & Control $(n / N)$ & Weight (\%) & RR & $95 \% \mathrm{CI}$ \\
\hline \multicolumn{6}{|l|}{ (1) All-cause mortality } \\
\hline \multicolumn{6}{|c|}{ (1.1) CDDP + conventional therapy versus conventional therapy } \\
\hline Guo et al. 2010 [20] & $5 / 76$ & $5 / 60$ & 19.10 & 0.79 & {$[0.24,2.60]$} \\
\hline Li et al. 2011 [19] & $6 / 252$ & $4 / 248$ & 13.80 & 1.48 & {$[0.42,5.17]$} \\
\hline Ma $2010[35]$ & $5 / 78$ & $11 / 85$ & 36.10 & 0.50 & {$[0.18,1.36]$} \\
\hline $\mathrm{Xu}$ and Wang 2007 [21] & $3 / 66$ & $10 / 80$ & 31.00 & 0.36 & {$[0.10,1.27]$} \\
\hline Total $\left(\mathrm{FEM}, I^{2}=0 \%\right)$ & & & 100.00 & 0.65 & {$[0.37,1.14]$} \\
\hline \multicolumn{6}{|l|}{ Sensitive analysis } \\
\hline Guo et al. 2010 [20] & $5 / 76$ & $5 / 60$ & 22.20 & 0.79 & {$[0.24,2.60]$} \\
\hline Ma $2010[35]$ & $5 / 78$ & $11 / 85$ & 41.80 & 0.50 & {$[0.18,1.36]$} \\
\hline $\mathrm{Xu}$ and Wang 2007 [21] & $3 / 66$ & $10 / 80$ & 35.90 & 0.36 & {$[0.10,1.27]$} \\
\hline Total $\left(\mathrm{FEM}, \mathrm{I}^{2}=\mathbf{0} \%\right)$ & & & 100.00 & 0.51 & {$[0.27,0.98]$} \\
\hline \multicolumn{6}{|c|}{ (1.2) CDDP + conventional therapy versus propranolol + conventional therapy } \\
\hline Xu and Wang 2007 [21] & $3 / 66$ & $5 / 72$ & 100.00 & 0.65 & {$[0.16,2.63]$} \\
\hline \multicolumn{6}{|l|}{ (2) Cardiac mortality } \\
\hline \multicolumn{6}{|c|}{ (2.1) CDDP + conventional therapy versus conventional therapy } \\
\hline Ma $2010[35]$ & $5 / 78$ & $11 / 85$ & 53.80 & 0.50 & {$[0.18,1.36]$} \\
\hline Xu and Wang 2007 [21] & $3 / 66$ & $10 / 80$ & 46.20 & 0.36 & {$[0.10,1.27]$} \\
\hline Total $\left(\right.$ FEM, $\left.\mathbf{I}^{2}=0 \%\right)$ & & & 100.00 & 0.43 & {$[0.20,0.95]$} \\
\hline \multicolumn{6}{|c|}{ (2.2) CDDP + conventional therapy versus placebo + conventional therapy } \\
\hline Li et al. 2010 [36] & $1 / 42$ & $1 / 21$ & 100.00 & 0.50 & {$[0.03,7.60]$} \\
\hline \multicolumn{6}{|c|}{ (2.3) CDDP + conventional therapy versus propranolol + conventional therapy } \\
\hline Xu and Wang 2007 [21] & $3 / 66$ & $4 / 72$ & 100.00 & 0.81 & {$[0.17,3.76]$} \\
\hline \multicolumn{6}{|c|}{ (3) Recurrent myocardial infarction } \\
\hline \multicolumn{6}{|c|}{ (3.1) CDDP + conventional therapy versus conventional therapy } \\
\hline Xu and Wang 2007 [21] & $2 / 66$ & $8 / 80$ & 100.00 & 0.30 & {$[0.07,1.38]$} \\
\hline \multicolumn{6}{|c|}{ (3.2) CDDP + conventional therapy versus placebo + conventional therapy } \\
\hline Li et al. 2010 [36] & $3 / 42$ & $3 / 21$ & 100.00 & 0.50 & {$[0.11,2.27]$} \\
\hline \multicolumn{6}{|c|}{ (3.3) CDDP + conventional therapy versus propranolol + conventional therapy } \\
\hline $\mathrm{Xu}$ and Wang 2007 [21] & $2 / 66$ & $3 / 72$ & 100.00 & 0.73 & {$[0.13,4.22]$} \\
\hline
\end{tabular}

bias is presented in Figure 4. The quality of evidence in the main comparison (CDDP versus no intervention) was low (Table 4).

3.4.4. Heart Failure (Table 6). Four studies [19-21, 36] reported heart failure in three different comparisons. Metaanalysis of three studies [19-21] found that CDDP was associated with a statistically significant reduction in the risk of heart failure compared with no intervention with no heterogeneity (RR $0.41 ; 95 \%$ CI 0.22 to $0.75 ; n=782 ; I^{2}=0 \%$ ). Sensitive analysis, excluding the lower quality study [19], got a similar conclusion (RR 0.30 ; $95 \%$ CI 0.14 to 0.65 ; two studies, $\left.n=282 ; I^{2}=0 \%\right)[20,21]$. Compared with propranolol on the basis of conventional therapy, CDDP still presented a statistical difference in reducing heart failure (RR 0.26; 95\%CI 0.07 to 0.99 ; one study, $n=138$ ) [21]. Nevertheless, compared with placebo on the basis of conventional therapy, CDDP showed no effect in the reduction of heart failure (RR 0.63; 95\%CI 0.19 to 2.09; one study, $n=63$ ) [36]. The associated risk of bias is presented in Figure 5. And the quality of evidence in the main comparison (CDDP versus no intervention) was moderate (Table 4 ).

3.4.5. Recurrent Angina (Table 6). Three studies [19, 20, 36] assessed the number of patients having recurrent angina in two different comparisons. While meta-analysis of two studies showed that CDDP was associated with a statistically significant reduction in the risk of recurrent angina compared with no intervention; the heterogeneity was significant (RR $0.43 ; 95 \%$ CI 0.29 to $\left.0.64 ; n=636 ; I^{2}=61 \%\right)[19,20]$. We, hence, examined the data and looked over the papers carefully. We found that besides the types of conventional therapy, the sample sizes between the two studies were also of big differences. Furthermore, one study was high risk of bias [19]. Random effects model, therefore, was used and got a different result without statistical difference (RR 0.33; 95\%CI 0.10 to $1.03 ; n=636$ ). Compared with placebo on the basis of conventional therapy, CDDP still showed no effect in the reduction of recurrent angina (RR 0.55 ; 95\% CI 0.29 to 1.02 ; one study, $n=63$ ) [36]. Figure 6 presents the associated 
TABLE 6: Analyses of secondary outcomes.

\begin{tabular}{|c|c|c|c|c|c|}
\hline Outcomes (comparisons) & Treatment $(n / N)$ & Control $(n / N)$ & Weight (\%) & $\mathrm{RR}$ & $95 \% \mathrm{CI}$ \\
\hline \multicolumn{6}{|l|}{ (1) Heart failure } \\
\hline \multicolumn{6}{|c|}{ (1.1) CDDP + conventional therapy versus conventional therapy } \\
\hline Xu and Wang 2007 [21] & $3 / 66$ & $12 / 80$ & 32.40 & 0.30 & {$[0.09,1.03]$} \\
\hline Guo et al. 2010 [20] & $5 / 76$ & $13 / 60$ & 43.40 & 0.30 & {$[0.11,0.80]$} \\
\hline Li et al. 2011 [19] & $6 / 252$ & $8 / 248$ & 24.10 & 0.74 & {$[0.26,2.10]$} \\
\hline Total $\left(\mathrm{FEM}, \mathrm{I}^{2}=\mathbf{0} \%\right)$ & & & 100.00 & 0.41 & {$[0.22,0.75]$} \\
\hline \multicolumn{6}{|l|}{ Sensitive analysis } \\
\hline $\mathrm{Xu}$ and Wang 2007 [21] & $3 / 66$ & $12 / 80$ & 57.30 & 0.30 & {$[0.09,1.03]$} \\
\hline Guo et al. 2010 [20] & $5 / 76$ & $13 / 60$ & 42.70 & 0.30 & {$[0.11,0.80]$} \\
\hline Total $\left(\right.$ FEM, $\left.\mathbf{I}^{2}=\mathbf{0} \%\right)$ & & & 100.00 & 0.30 & {$[0.14,0.65]$} \\
\hline \multicolumn{6}{|c|}{ (1.2) CDDP + conventional therapy versus placebo + conventional therapy } \\
\hline Li et al. 2010 [36] & $5 / 42$ & $4 / 21$ & 100.00 & 0.63 & {$[0.19,2.09]$} \\
\hline \multicolumn{6}{|c|}{ (1.3) CDDP + conventional therapy versus propranolol + conventional therapy } \\
\hline $\mathrm{Xu}$ and Wang 2007 [21] & $3 / 66$ & $11 / 72$ & 100.00 & 0.26 & {$[0.07,0.99]$} \\
\hline \multicolumn{6}{|l|}{ (2) Recurrent angina } \\
\hline \multicolumn{6}{|c|}{ (2.1) CDDP + conventional therapy versus conventional therapy } \\
\hline Guo 2010 [20] & $2 / 76$ & $11 / 60$ & 33.40 & 0.14 & {$[0.03,0.62]$} \\
\hline Li et al. $2011[19]$ & $27 / 252$ & $54 / 248$ & 66.60 & 0.49 & {$[0.32,0.75]$} \\
\hline Total $\left(\right.$ REM, $\left.I^{2}=61 \%\right)$ & & & 100.00 & 0.33 & {$[0.10,1.03]$} \\
\hline \multicolumn{6}{|c|}{ (2.2) CDDP + conventional therapy versus placebo + conventional therapy } \\
\hline Li et al. $2010[36]$ & $12 / 42$ & $11 / 21$ & 100.00 & 0.55 & {$[0.29,1.02]$} \\
\hline \multicolumn{6}{|l|}{ (3) Readmission } \\
\hline \multicolumn{6}{|c|}{ CDDP + conventional therapy versus placebo + conventional therapy } \\
\hline Li et al. 2010 [36] & $3 / 42$ & $4 / 21$ & 100.00 & 0.38 & {$[0.09,1.52]$} \\
\hline
\end{tabular}

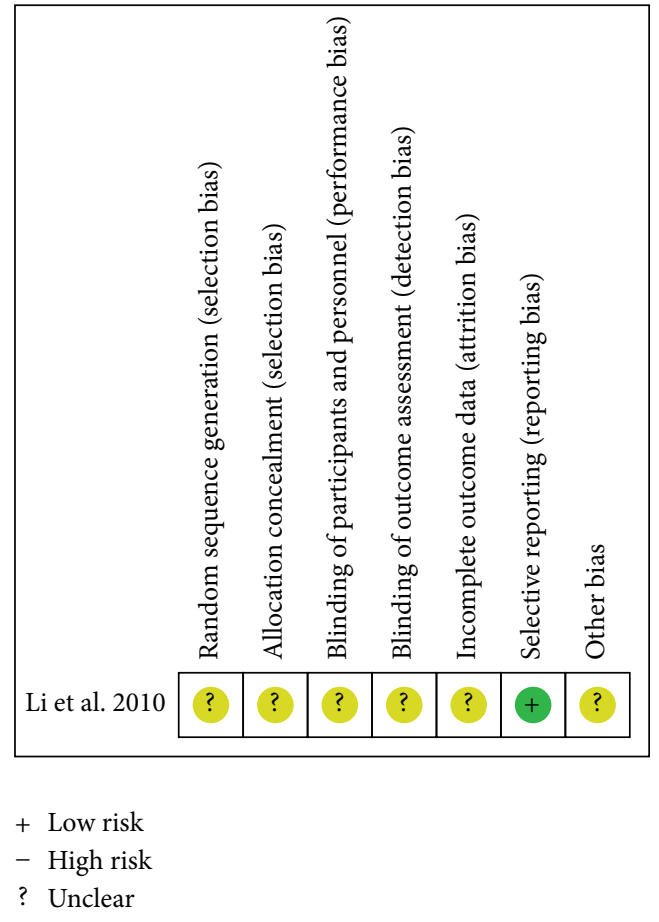

FIGURE 8: Risk of bias summary-QOL. risk of bias. The quality of evidence in the main comparison (CDDP versus no intervention) was low (Table 4).

3.4.6. Readmission (Table 6). Only one study reported readmission in the comparison of CDDP plus conventional therapy versus placebo plus conventional therapy (RR 0.38; $95 \%$ CI 0.09 to $1.52 ; n=63$ ) [36]. The associated risk of bias is presented in Figure 7.

3.4.7. Quality of Life (Table 7). One study assessed QOL by questionnaire score. The questionnaire was designed referring to TOMHS and SF-36. Compared with placebo group on the basis of conventional therapy, patients in the group treated with CDDP had higher scores (MD 12.60; 95\%CI 3.23 to $21.97 ; n=63$ ) [36]. The associated risk of bias is presented in Figure 8.

3.4.8. Left Ventricular Ejection Fraction (Table 7). Five studies $[19,21,33,34,36]$ assessed LVEF in three different comparisons. Meta-analysis (random effects model) of four studies $[19,21,33,34]$ found that CDDP was associated with a statistically significant increase in LVEF compared with no intervention (MD 4.79\%; 95\%CI 3.31 to $6.28 ; n=$ $781)$. For the significant heterogeneity $\left(I^{2}=51 \%\right)$ among 
TABLE 7: Analyses of secondary outcomes.

\begin{tabular}{|c|c|c|c|c|c|c|c|c|c|}
\hline \multirow{2}{*}{ Outcomes (comparisons) } & \multicolumn{3}{|c|}{ Treatment } & \multicolumn{3}{|c|}{ Control } & \multirow{2}{*}{ Weight (\%) } & \multirow{2}{*}{ MD } & \multirow{2}{*}{$95 \%$ CI } \\
\hline & Mean & SD & $N$ & Mean & SD & $N$ & & & \\
\hline \multicolumn{10}{|l|}{ (4) $L V E F \%$} \\
\hline \multicolumn{10}{|c|}{ (4.1) CDDP + conventional therapy versus conventional therapy } \\
\hline Mei et al. 2006 [34] & 60.80 & 7.20 & 23 & 59.20 & 6.80 & 22 & 10.50 & 1.60 & {$[-2.49,5.69]$} \\
\hline Xu and Wang 2007 [21] & 51.20 & 4.30 & 66 & 47.10 & 4.60 & 80 & 34.60 & 4.10 & {$[2.65,5.55]$} \\
\hline Li et al. 2011 [19] & 57.10 & 8.70 & 252 & 51.90 & 9.90 & 248 & 31.70 & 5.20 & {$[3.57,6.83]$} \\
\hline Lin $2011[33]$ & 54.50 & 6.80 & 46 & 47.80 & 3.90 & 44 & 23.30 & 6.70 & {$[4.42,8.98]$} \\
\hline Total $\left(\mathrm{REM}, \mathrm{I}^{2}=51 \%\right)$ & & & & & & & 100.00 & 4.79 & {$[3.31,6.28]$} \\
\hline \multicolumn{10}{|c|}{ Subgroup analysis (according to duration of treatment) } \\
\hline \multicolumn{10}{|l|}{ (4.1.1) 30 days -6 weeks } \\
\hline Li et al. 2011 [19] & 57.10 & 8.70 & 252 & 51.90 & 9.90 & 248 & 33.90 & 5.20 & {$[3.57,6.83]$} \\
\hline Lin 2011 [33] & 54.50 & 6.80 & 46 & 47.80 & 3.90 & 44 & 17.40 & 6.70 & {$[4.42,8.98]$} \\
\hline Subtotal $\left(\mathrm{FEM}, \mathrm{I}^{2}=9 \%\right)$ & & & & & & & 51.30 & 5.71 & {$[4.38,7.04]$} \\
\hline \multicolumn{10}{|l|}{ (4.1.2) 6 months -12 months } \\
\hline Mei et al. 2006 [34] & 60.80 & 7.20 & 23 & 59.20 & 6.80 & 22 & 5.40 & 1.60 & {$[-2.49,5.69]$} \\
\hline $\mathrm{Xu}$ and Wang 2007 [21] & 51.20 & 4.30 & 66 & 47.10 & 4.60 & 80 & 43.30 & 4.10 & {$[2.65,5.55]$} \\
\hline Subtotal (FEM, I $\left.{ }^{2}=22 \%\right)$ & & & & & & & 48.70 & 3.82 & {$[2.46,5.19]$} \\
\hline \multicolumn{10}{|c|}{ (4.2) CDDP + conventional therapy versus placebo + conventional therapy } \\
\hline Li et al. $2010[36]$ & 55.69 & 9.34 & 42 & 50.21 & 7.83 & 21 & 100.00 & 5.48 & {$[1.10,9.86]$} \\
\hline \multicolumn{10}{|c|}{ (4.3) CDDP + conventional therapy versus propranolol + conventional therapy } \\
\hline Xu and Wang 2007 [21] & 51.20 & 4.30 & 66 & 49.60 & 5.00 & 72 & 100.00 & 1.60 & {$[0.05,3.15]$} \\
\hline \multicolumn{10}{|l|}{ (5) Quality of life (score) } \\
\hline \multicolumn{10}{|c|}{ CDDP + conventional therapy versus placebo + conventional therapy } \\
\hline Li et al. 2010 [36] & 110.28 & 19.33 & 42 & 97.68 & 17.13 & 21 & 100.00 & 12.60 & {$[3.23,21.97]$} \\
\hline
\end{tabular}

the studies, we examined the data and looked over the papers carefully. We found that there was a significant difference in the duration of treatment among the studies. Therefore, we conducted a subgroup analysis according to the duration of treatment. In the subgroup analysis of patients with 30 days to six weeks treatment $[19,33]$ versus six months to 12 months treatment $[21,34]$, the test effect still had statistical significant but without significant heterogeneity: MD 5.71\% (95\%CI 4.38 to 7.04 ; two studies, $n=590 ; I^{2}=9 \%$ ) for 30 days to six weeks treatment versus MD 3.82\% (95\%CI 2.46 to 5.19; two studies, $\left.n=191 ; I^{2}=22 \%\right)$ for six months to 12 months treatment. Compared with placebo on the basis of conventional therapy, CDDP also presented a statistical difference in the increase of LVEF (MD 5.48\%; 95\%CI 1.10 to 9.86; one study, $n=63$ ) [36]. In addition, a single study reported a similar result between CDDP and propranolol (MD 1.60\%; 95\%CI 0.05 to $3.15 ; n=$ 138) [21]. Figure 9 presents the associated risk of bias. The quality of evidence in the main comparison (CDDP versus no intervention) was low (Table 4).

3.4.9. Adverse Events. One of the seven studies reported adverse events [36]. The authors described that there were mild adverse events in the CDDP group such as blushing (1/63 patient), abdominal distention (2/63 patients), dizziness, and distention of head (2/63 patients). However, all of the adverse events remitted spontaneously. There were no significant differences between the two groups in blood glucose, hepatic function, and renal function after treatment. The associated risk of bias is presented in Figure 10.

\section{Discussion}

Seven RCTs including 1215 participants were included in this review. CDDP presented statistically significant benefit on the incidence of cardiac death and heart failure as compared with no intervention based on conventional therapy for AMI. Compared with propranolol, CDDP showed the similar effect on heart failure. In addition, the benefit of CDDP on LVEF was statistically significant both in short-term (30 days to six weeks) and long-term (six months to 12 months) treatment compared with no intervention, placebo, or propranolol. CDDP was also associated with a statistically significant improvement in QOL compared with placebo on the basis of conventional therapy. However, it was not associated with a statistically significant effect on RMI, readmission, or recurrent angina. Unfortunately, no data was available to assess the effect of CDDP on revascularization.

The discrepancy between the effect on all-cause mortality before and after sensitive analysis might be related to the lower quality study [19]. Although CDDP was found to be beneficial for the reduction of all-cause mortality after sensitive analysis, the effect still need to be demonstrated due to the low quality of the evidence. 


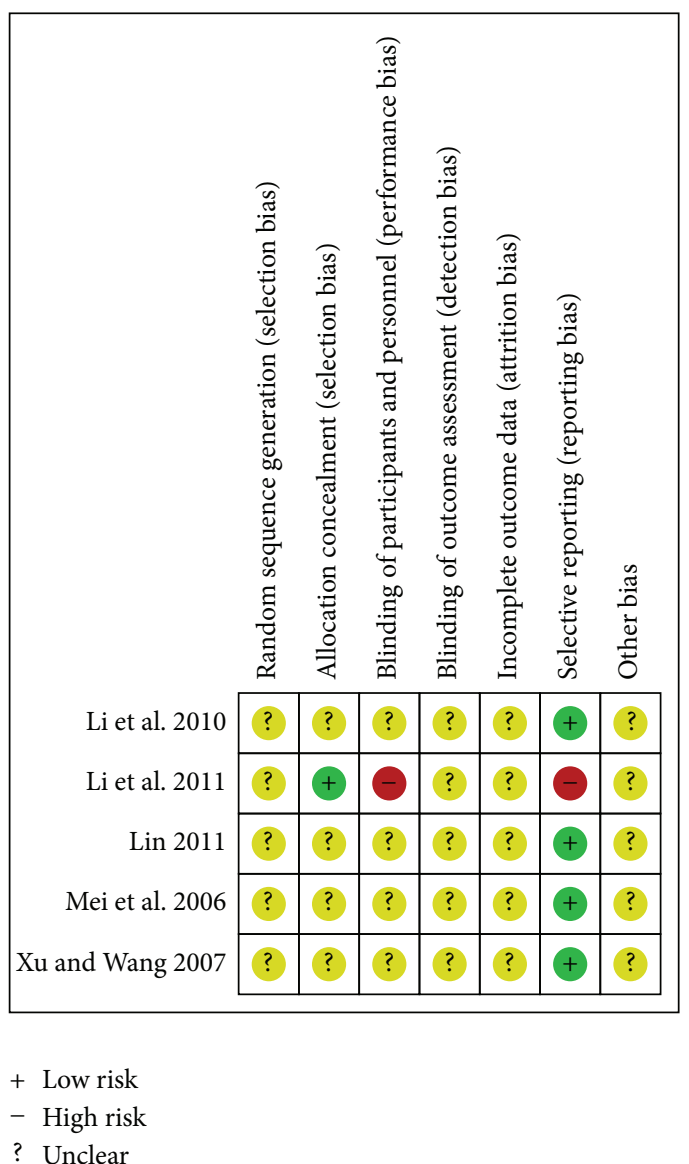

FIGURE 9: Risk of bias summary-LVEF.

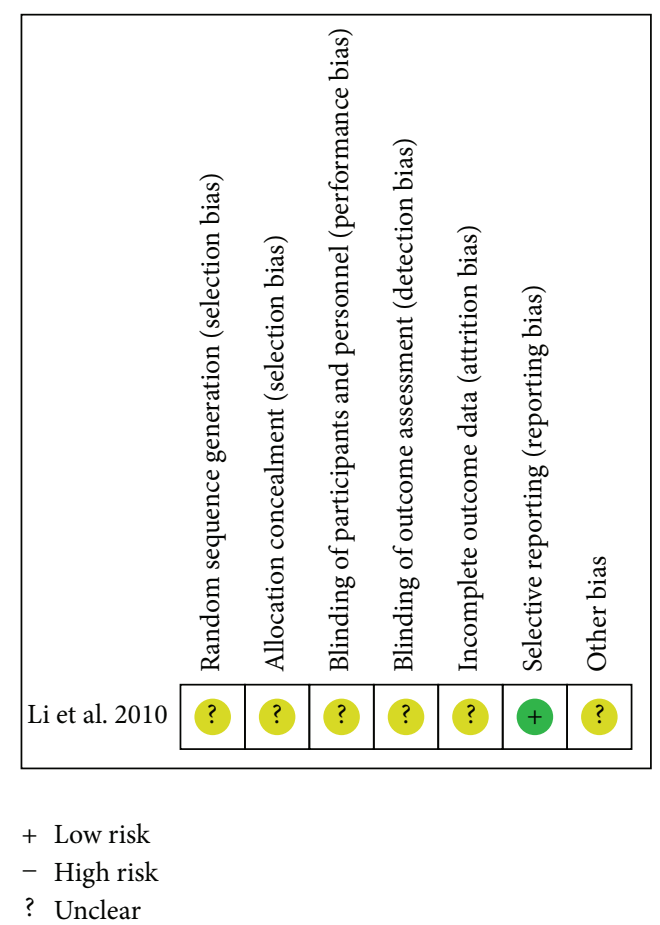

FIGURE 10: Risk of bias summary—adverse events.
When we mention TCM, often natural products with fewer side effects come to mind. In fact, systematic reviews [22-24] do indicate fewer mild side effects of CDDP for angina pectoris. A latest parallel double blind randomized placebo-controlled trial also showed no significant adverse effects of CDDP for hypercholesterolemia patients [9]. However, in this review, only one study with small simple size described mild adverse events of CDDP with spontaneous remission. Due to the insufficient data, it is too early to evaluate the safety of CDDP for AMI patients at present. We, therefore, suggest detailed description of adverse events in the future studies of CDDP.

We have to consider a number of limitations in this review before recommending the conclusion to clinical practitioners. (1) We might miss some unpublished relevant studies since we only searched unpublished studies from CPCD, CDFD, and CMFD. What is more, we could not create a funnel plot to check for possible publication bias for each outcome due to the low number of included studies. Publication bias might exist in our results. (2) None of the included studies was assessed to be at low risk of bias. The main reasons are as follows: firstly, the method of random sequence generation was unclear in most of the studies, and only one study reported allocation concealment; most of the studies might have selection bias; secondly, no study described double blind method as well as the blinding of outcome assessment; both selection bias and detection bias might exist in the conclusion; thirdly, neither withdrawals nor losses to follow up was reported in each study; this could lead to a high risk of attrition bias; fourthly, one study [19] had selective reporting on cardiac mortality and RMI which should be reported in accordance with its study plan; this could induce reporting bias. In addition, all of the included studies did not mention ITT analysis, which might lead to some other bias. (3) Most of the durations of follow up were short; the reliability and validity of some outcomes such as mortality could be influenced. (4) The small number of included studies and the different comparisons among the studies precluded us from conducting subgroup analyses to explore effect modifiers such as duration of intervention and type of conventional therapy. (5) For some outcomes, only single study provided data and most of the studies did not meet the calculated optimal information size. This might influence the precision of results, which could downgrade the quality of evidence. (6) We assessed the quality of evidence for each outcome according to the GRADE approach with caution. However, the overall quality of evidence in the main comparison was poor, which can weaken the strength of recommendation.

Although this systematic review suggests some benefits of CDDP for AMI patients, the recommendation of findings was limited due to the poor quality studies. Therefore, rigorously designed clinical trials are warranted to further demonstrate the effectiveness and safety of CDDP for AMI. Moreover, we suggest that researchers of RCTs provide complete, clear, and transparent information on their methodologies and findings in the future. This is important for readers or reviewers to assess and use RCTs accurately. Thus, we expect that more RCTs of TCM will be appropriately designed, conducted, and 
reported according to the CONSORT statement [37] or the CONSORT statement for herbal interventions [38].

\section{Conclusion}

This systematic review found the following potential benefits from CDDP added to conversional therapy in AMI patients: reduction of cardiac death and heart failure, improvement of QOL and LVEF. However, the benefits should be considered due to the poor quality of evidence. In addition, the safety of CDDP has not been confirmed for the deficiency of available studies. More high quality evidence from high quality RCTs is needed to support the clinical use of CDDP for AMI patients.

\section{Conflict of Interests}

The authors declare no conflict of interests.

\section{Authors' Contribution}

J. Luo developed the search strategy and data extraction form, searched and identified trials, extracted data and analyzed data, and drafted the paper. H. Xu conceived and designed the study, helped with development of the search strategy, identified trials and extracted data, verified data analyses, and revised the paper. K. J. Chen conceived and designed the study, provided methodological perspectives, and revised the paper.

\section{Acknowledgments}

The current work was supported by the National Key Basic Research Program of China (no. 2006CB504803) and the Twelve Five-year Plan of China (nos. 2013BAI02B01 and 2013BAI13B01). The authors thank Jun Xia, researcher of Cochrane Schizophrenia Group, for her kind help in the writing of this review and Stephanie Sampson, researcher of Cochrane Schizophrenia Group, and Yaolong Chen, researcher of Chinese GRADE Center, for advice on the quality assessment in the review.

\section{References}

[1] H. D. White and D. P. Chew, "Acute myocardial infarction," The Lancet, vol. 372, no. 9638, pp. 570-584, 2008.

[2] P. Libby, "Current concepts of the pathogenesis of the acute coronary syndromes," Circulation, vol. 104, no. 3, pp. 365-372, 2001.

[3] X.-J. Ma, H.-J. Yin, and K.-J. Chen, "Appraisal of the prognosis in patients with acute myocardial infarction treated with primary percutaneous coronary intervention," Chinese Journal of Integrative Medicine, vol. 15, no. 3, pp. 236-240, 2009.

[4] K. J. Chen, K. K. Hui, M. S. Lee, and H. Xu, "The potential benefit of complementary/alternative medicine in cardiovascular diseases," Evidence-Based Complementary and Alternative Medicine, vol. 2012, Article ID 125029, 1 page, 2012.

[5] G. Dobos and I. Tao, "The model of Western integrative medicine: the role of Chinese medicine," Chinese Journal of Integrative Medicine, vol. 17, no. 1, pp. 11-20, 2011.
[6] A. S. Ferreira and A. J. Lopes, "Chinese medicine pattern differentiation and its implications for clinical practice," Chinese Journal of Integrative Medicine, vol. 17, no. 11, pp. 818-823, 2011.

[7] H. Xu and K.-J. Chen, "Integrating traditional medicine with biomedicine towards a patient-centered healthcare system," Chinese Journal of Integrative Medicine, vol. 17, no. 2, pp. 83-84, 2011.

[8] K.-J. Chen and A.-P. Lu, "Situation of integrative medicine in China: results from a National Survey in 2004," Chinese Journal of Integrative Medicine, vol. 12, no. 3, pp. 161-165, 2006.

[9] K. A. O’Brien, S. H. Ling, E. Abbas et al., "A Chinese herbal preparation containing radix salviae miltiorrhizae, radix notoginseng and borneolum syntheticum reduces circulating adhesion molecules," Evidence-based Complementary and Alternative Medicine, vol. 2011, Article ID 790784, 6 pages, 2011.

[10] X.-Y. Ji, B. K.-H. Tan, and Y.-Z. Zhu, "Salvia miltiorrhiza and ischemic diseases," Acta Pharmacologica Sinica, vol. 21, no. 12, pp. 1089-1094, 2000.

[11] C. D. Wing-Shing, C. M. Koon, C. F. Ng et al., "The roots of Salvia miltiorrhiza (Danshen) and Pueraria lobata (Gegen) inhibit atherogenic events: a study of the combination effects of the 2-herb formula," Journal of Ethnopharmacology, vol. 143, no. 3, pp. 859-866, 2012.

[12] J. Leng, C. M. Fu, and F. Wan, "Research progress on chemical compositions and pharmacological effects of panaxatriol saponins," West China Journal of Pharmaceutical Sciences, vol. 26, no. 1, pp. 83-86, 2011.

[13] Z. G. Yang, A. Q. Chen, and S. D. Yu, "Research progress of pharmacological actions of Panax notoginseng," Shanghai Journal of Traditional Chinese Medicine, vol. 39, no. 4, pp. 5962, 2005.

[14] S. R. Wu, G. Cheng, and Y. Feng, "Progress in studies on pharmacology of borneol," Chinese Traditional and Herbal Drugs, vol. 32, no. 12, pp. 90-92, 2001.

[15] Y. Liu, B. L. Zhang, and L. M. Hu, "General view on pharmacological research of borneol," Tianjin Journal of Traditional Chinese Medicine, vol. 20, no. 4, pp. 85-87, 2003.

[16] Y. Lu and M. J. Jia, "Pharmacological and clinical research of Compound Danshen Dropping Pill in coronary heart disease," Chinese Heart Journal, vol. 12, no. 5, pp. 418-419, 2000.

[17] J. Sun, S. H. Huang, B. K.-H. Tan et al., "Effects of purified herbal extract of Salvia miltiorrhiza on ischemic rat myocardium after acute myocardial infarction," Life Sciences, vol. 76, no. 24, pp. 2849-2860, 2005.

[18] X. Ji, B. K.-H. Tan, Y. C. Zhu, W. Linz, and Y. Z. Zhu, "Comparison of cardioprotective effects using ramipril and DanShen for the treatment of acute myocardial infarction in rats," Life Sciences, vol. 73, no. 11, pp. 1413-1426, 2003.

[19] G. P. Li, X. T. Zheng, H. Z. Wang et al., "Multicenter investigation of Compound Danshen Dripping Pills on short-term clinical events in patient with ST elevation myocardial infarction undergoing primary PCI, (MICD-STEMIPCI)," Chinese Journal of Interventional Cardiology, vol. 19, no. 1, pp. 24-28, 2011.

[20] H. Y. Guo, S. F. Lin, and X. Z. Kang, "Effects of Compound Danshen Dripping Pills on reperfusion injury after intravenous thrombolytic therapy in acute myocardial infarction," Journal of Chinese Physician, vol. 38, no. 7, pp. 49-50, 2010.

[21] H. Xu and J. Wang, "Effects of Compound Danshen Dripping Pill on survival rate of patients with acute myocardial infarction," Proceeding of Clinical Medicine, vol. 16, no. 6, pp. 429-430, 2007. 
[22] Y. M. Guo, C. Zhang, Q. L. Cha et al., "Compound Salvia Droplet Pill for treatment of coronary heart disease: a systematic review," Journal of Shanghai University of Traditional Chinese Medicine, vol. 26, no. 3, pp. 24-31, 2012.

[23] J. Zhang, M.-Z. Zhang, and L. Wang, "The systematic review of compoud Danshen dripping pills in treatment of coronary heart disease," Chinese Journal of New Drugs, vol. 18, no. 6, pp. 465-468, 2009.

[24] W. Chen, L. M. Qin, Z. H. Liu, and Z. R. Xu, “The systematic review of Compound Danshen Dropping Pills in treatment of stable angina pectoris," Journal of Shandong University of Traditional Chinese Medicine, vol. 36, no. 4, pp. 287-291, 2012.

[25] Chinese Society of Cardiology of Chinese Medical Association and Editorial Board of Chinese Journal of Cardiology, "Guideline for the diagnosis and treatment of patients with acute myocardial infarction," Chinese Journal of Cardiology, vol. 29, no. 12, pp. 9-24, 2001.

[26] E. Braunwald, E. M. Antman, J. W. Beasley et al., "ACC/AHA guidelines for the management of patients with unstable angina and non-ST-segment elevation myocardial infarction: executive summary and recommendations: a report of the American College of Cardiology/American Heart Association task force on practice guidelines (committee on the management of patients with unstable angina)," Circulation, vol. 102, no. 10, pp. 1193-1209, 2000.

[27] Chinese Society of Cardiology of Chinese Medical Association and Editorial Board of Chinese Journal of Cardiology, "Guideline for diagnosis and treatment of patients with unstable angina and non-ST-segment elevation myocardial infarction," Chinese Journal of Cardiology, vol. 35, no. 4, pp. 295-304, 2007.

[28] Chinese Society of Cardiology of Chinese Medical Association and Editorial Board of Chinese Journal of Cardiology, "Recommendation of the application of universal definition of myocardial infarction in China," Chinese Journal of Cardiology, vol. 36, no. 10, pp. 867-869, 2008.

[29] Chinese Society of Cardiology of Chinese Medical Association and Editorial Board of Chinese Journal of Cardiology, "Guideline for diagnosis and treatment of patients with ST-elevation myocardial infarction," Chinese Journal of Cardiology, vol. 38, no. 8, pp. 675-687, 2010.

[30] J. P. T. Higgins and S. Green, Cochrane Handbook for Systematic Reviews of Interventions, Version 5.1.0, The Cochrane Collaboration, 2011, http://handbook.cochrane.org/.

[31] J. Brozek, A. Oxman, and H. Schünemann, "GRADEpro. [Computer program]. Version 3.2 for Windows," 2008, http://ims. cochrane.org/revman/other-resources/gradepro.

[32] J. P. T. Higgins, S. G. Thompson, J. J. Deeks, and D. G. Altman, "Measuring inconsistency in meta-analyses," British Medical Journal, vol. 327, no. 7414, pp. 557-560, 2003.

[33] X. D. Lin, "Clinical trial of Compound Danshen Dripping Pill on ventricular remodeling after acute myocardial infarction," World Chinese Medicine, vol. 6, no. 2, pp. 111-112, 2011.

[34] F. G. Mei, Y. Q. Zhang, Z. L. Wang, and G. Zhao, "Effects of Compound Danshen Dripping Pill on BNP and left ventricular function in patients with acute myocardial infarction," Heilongjiang Journal of Traditional Chinese Medicine, vol. 6, pp. 4-6, 2006.

[35] Y. H. Ma, Relationship Between Serum Level of Tumor Necrosis Factor-Alpha, Matrix Metalloproteinase-9 and Interleukin-6 and Clinical Prognosis and the Effects of Compound Danshen Dripping Pill on STEMI, Tianjin Medical University, Tianjin, China, 2010.
[36] X. F. Li, F. Liu, T. L. Jiang, Z. J. Wang, and Z. H. Wu, "Efficacy of compound Danshen drop pill 42 patients with early acute myocardial infarction," Chinese Journal of New Drugs, vol. 19, no. 18, pp. 1699-1702, 2010.

[37] K. F. Schulz, D. G. Altman, and D. Moher, “CONSORT 2010 statement: updated guidelines for reporting parallel group randomized trials," Annals of Internal Medicine, vol. 152, no. 11, pp. 726-732, 2010.

[38] J. J. Gagnier, H. Boon, P. Rochon, D. Moher, J. Barnes, and C. Bombardier, "Reporting randomized, controlled trials of herbal interventions: an elaborated CONSORT statement," Annals of Internal Medicine, vol. 144, no. 5, pp. 364-367, 2006. 


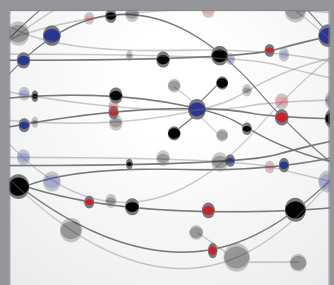

The Scientific World Journal
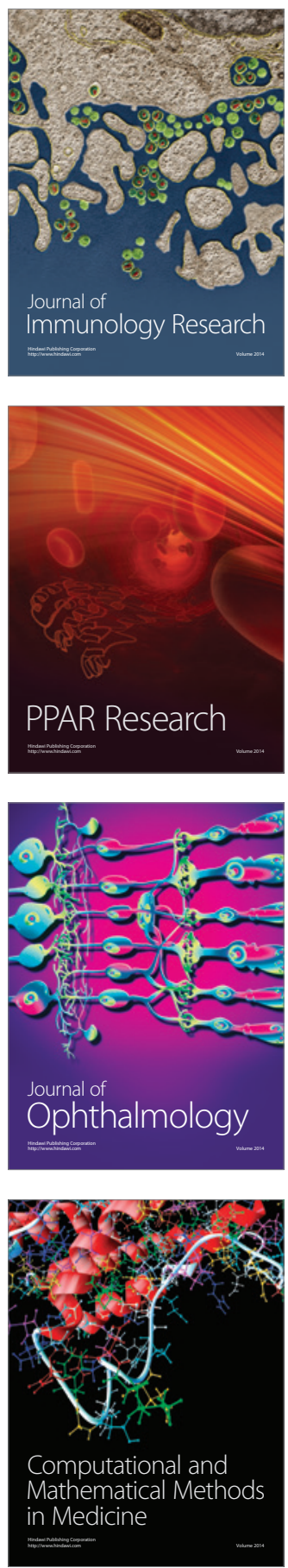

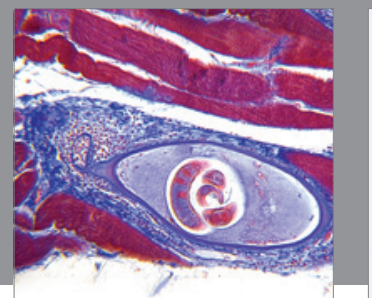

Gastroenterology

Research and Practice
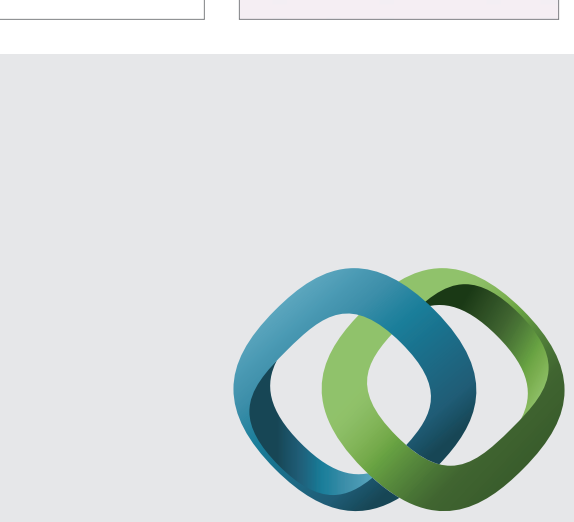

\section{Hindawi}

Submit your manuscripts at

http://www.hindawi.com
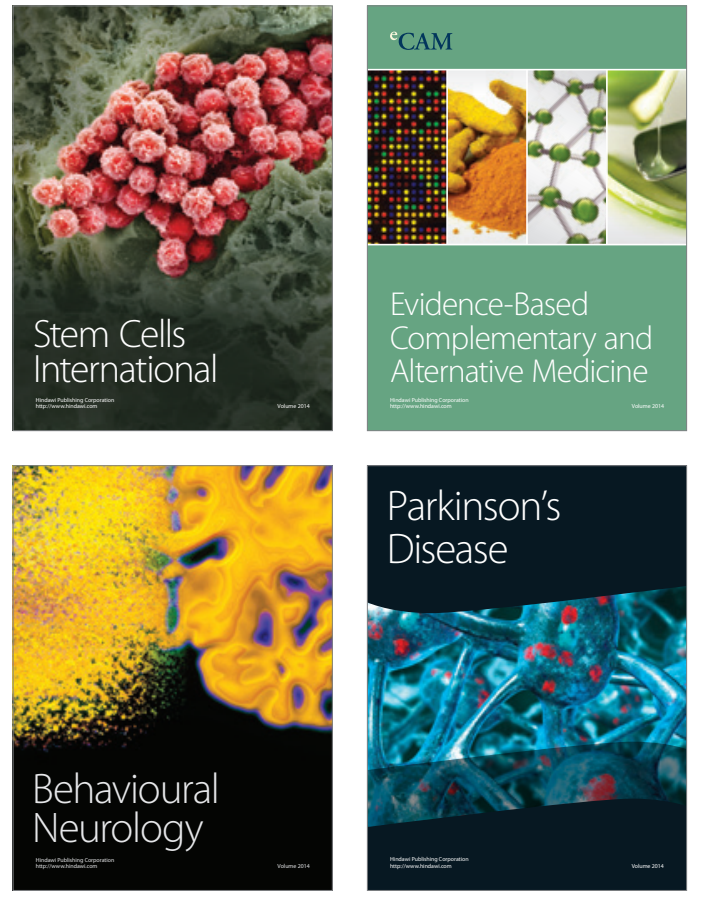
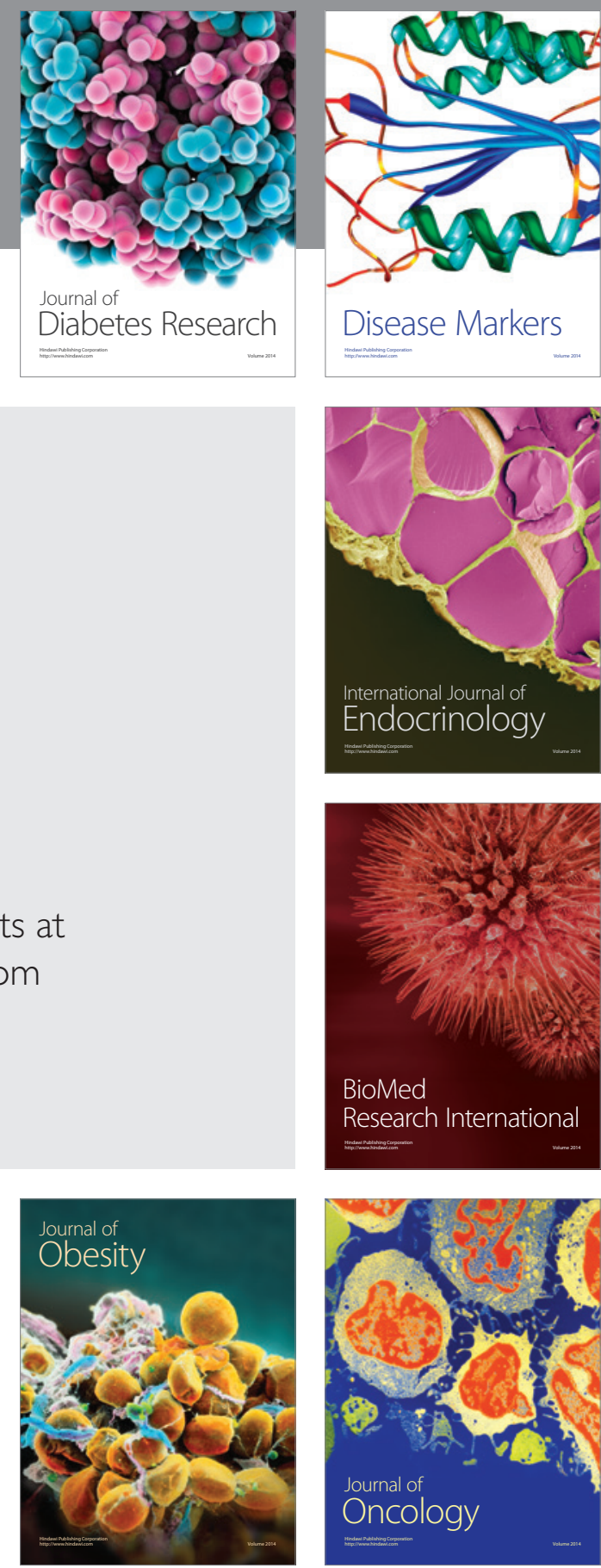

Disease Markers
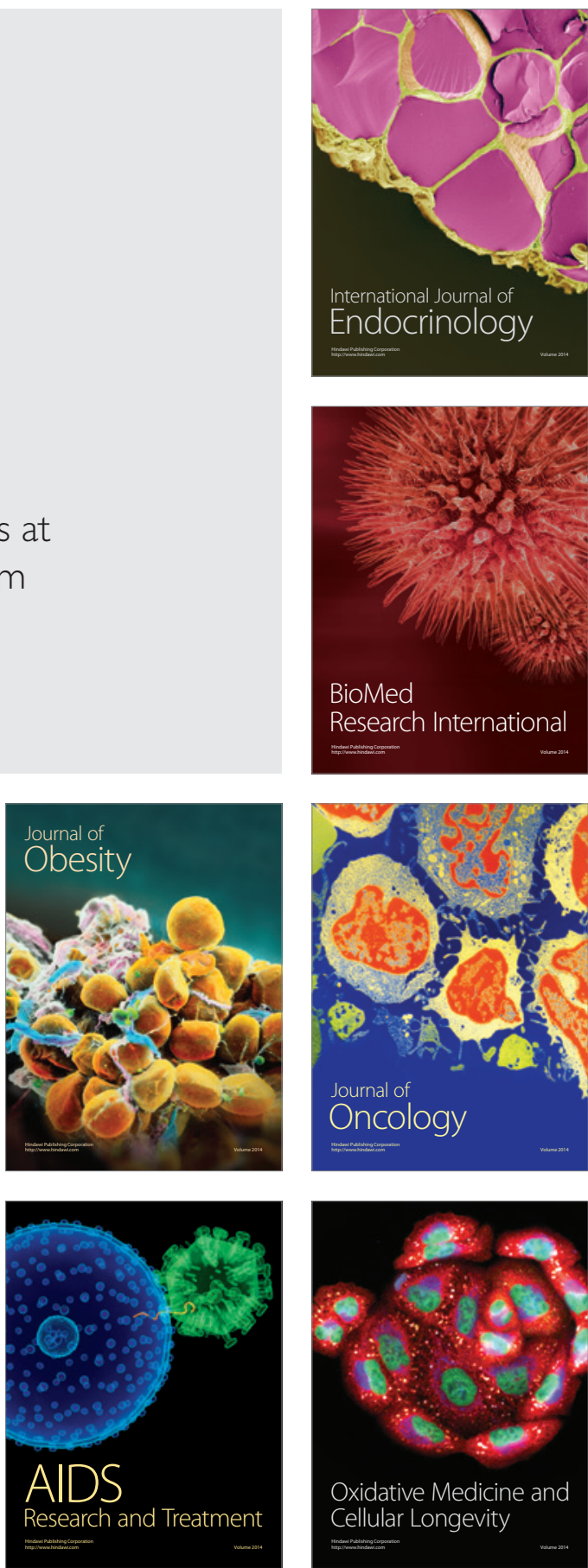